\title{
SXP 214: AN X-RAY PULSAR IN THE SMALL MAGELLANIC CLOUD, CROSSING THE CIRCUMSTELLAR
} DISK OF THE COMPANION

\author{
JaeSub Hong $^{1}$, Vallia Antoniou ${ }^{1}$, Andreas Zezas ${ }^{1,2,3}$, Frank Haberl ${ }^{4}$, Jeremy J. Drake ${ }^{1}$, Paul P. Plucinsky ${ }^{1}$, \\ Terrance Gaetz ${ }^{1}$, Manami Sasaki ${ }^{5}$, Benjamin Williams ${ }^{6}$, Knox S. Long ${ }^{7}$, William P. Blair ${ }^{8}$, P. Frank Winkler ${ }^{9}$, \\ Nicholas J. Wright ${ }^{10}$, Silas LaYCOCK ${ }^{11}$, AND ANDRZEJ UdALSKI ${ }^{12}$ \\ ${ }^{1}$ Harvard-Smithsonian Center for Astrophysics, 60 Garden St., Cambridge, MA 02138, USA; jaesub@ @ead.cfa.harvard.edu \\ ${ }^{2}$ Foundation for Research and Technology-Hellas, 71110 Heraklion, Crete, Greece \\ ${ }^{3}$ Physics Department \& Institute of Theoretical \& Computational Physics, University of Crete, 71003 Heraklion, Crete, Greece \\ ${ }^{4}$ Max-Planck-Institut für extraterrestrische Physik, Giessenbach straße, D-85748 Garching, Germany \\ ${ }^{5}$ Institut für Astronomie und Astrophysik, Universität Tübingen, Sand 1, D-72076, Tübingen, Germany \\ ${ }^{6}$ Department of Astronomy, Box 351580, University of Washington, Seattle, WA 98195, USA \\ 7 Space Telescope Science Institute, 3700 San Martin Drive, Baltimore, MD 21218, USA \\ ${ }^{8}$ The Henry A. Rowland Department of Physics and Astronomy, Johns Hopkins University, 3400 North Charles Street, Baltimore, MD 21218, USA \\ ${ }^{9}$ Department of Physics, Middlebury College, Middlebury, VT 05753, USA \\ ${ }^{10}$ Astrophysics Group, Keele University, Keele, ST5 5BG, UK \\ ${ }_{11}^{11}$ Department of Physics, University of Massachusetts Lowell, MA 01854, USA \\ ${ }^{12}$ Warsaw University Observatory, Al. Ujazdowskie 4, 00-478 Warszawa, Poland \\ Received 2016 February 7; revised 2016 May 9; accepted 2016 May 11; published 2016 July 14
}

\begin{abstract}
Located in the Small Magellanic Cloud (SMC), SXP 214 is an X-ray pulsar in a high mass X-ray binary system with a Be-star companion. A recent survey of the SMC under a Chandra X-ray Visionary program found that the source was in a transition when the X-ray flux was on a steady rise. The Lomb-Scargle periodogram revealed a pulse period of $211.49 \pm 0.42 \mathrm{~s}$, which is significantly $(>5 \sigma)$ shorter than the previous measurements made with $X M M-N e w t o n$ and RXTE. This implies that the system has gone through sudden spin-up episodes recently. The pulse profile shows a sharp eclipse-like feature with a modulation amplitude of $>95 \%$. The linear rise of the observed X-ray luminosity from $\lesssim 2 \times$ to $7 \times 10^{35} \mathrm{erg} \mathrm{s}^{-1}$ is correlated with a steady softening of the X-ray spectrum, which can be described by the changes in the local absorption from $N_{\mathrm{H}} \sim 10^{24}$ to $\lesssim 10^{20} \mathrm{~cm}^{-2}$ for an absorbed power-law model. The soft X-ray emission below $2 \mathrm{keV}$ was absent in the early part of the observation when only the pulsating hard X-ray component was observed, whereas at later times, both soft and hard X-ray components were observed to be pulsating. A likely explanation is that the neutron star was initially hidden in the circumstellar disk of the companion, and later came out of the disk with the accreted material that continued fueling the observed pulsation.
\end{abstract}

Key words: stars: neutron - X-rays: binaries

\section{INTRODUCTION}

The Small Magellanic Cloud (SMC) harbors a large number of high mass X-ray binaries with pulsating neutron stars (NSs) and Be-star companions (Coe \& Kirk 2015; Haberl \& Sturm 2015). In a system with a relatively long orbital period, the NS is often in a wide and eccentric orbit, and periodically encounters the circumstellar disk of the Be-star, which triggers Type I outbursts through accretion onto the NS (Reig 2011; Townsend et al. 2011). SXP 214 or XMMU J005011.2-730026 was discovered as a transient pulsar in the SMC with a pulse period of $P=214 \mathrm{~s}$ from an $X M M$ Newton observation in 2009 (Coe et al. 2011, hereafter C11). A B2-B3 III star with $V=15.3 \mathrm{mag}$ and a weak $\mathrm{H} \alpha$ emission line was identified as the optical counterpart from the OGLE-II and III data and an optical spectrum taken at the South African Astronomical Observatory (C11). Thus, SXP 214 has been classified as a Be/X-ray binary (Be-XRB; $\mathrm{C} 11)$.

The orbital period of the system remains unclear. C11 reported an optical periodicity of 4.52 days based on the OGLE-III light curve. Schmidtke \& Cowley (2011) reported another optical period of 0.82 days based on the MACHO and OGLE-II observations, arguing that the 4.52 day periodicity is a weaker alias of the 0.82 day period, and that the latter is more consistent with other Be-XRBs. Later, Schmidtke et al. (2013) reported a new optical period of 29.91 days with an eclipselike feature in the folded light curve from the OGLE-IV data when the optical counterpart became faint and the 0.82 day optical pulsations disappeared.

Here we describe new Chandra observations of SXP 214 (Section 2), which show dramatic temporal and spectral changes of the X-ray emission (Section 3). We search for periodicity in the X-ray emission and perform a phase-resolved spectral analysis to understand the origin of the observed changes (Section 4). We revisit the OGLE optical light curve and its long-term periodicity (Section 5). We present a likely scenario for the changes observed in the X-ray emission, and constrain the density of the circumstellar disk, the magnetic field of the NS, and the accretion rate (Section 6).

\section{OBSERVATIONS AND DATA PROCESSING}

SXP 214 was observed in 2013 January (Obs. ID 14670) and 2014 March (Obs. ID 15503) by Chandra for $50 \mathrm{ks}$ each as a part of the recent SMC survey under the Chandra X-ray Visionary Program (PI A. Zezas). The details of the survey program and the full source catalog are found in Antoniou et al. (2016, in preparation). The X-ray timing analysis and the catalog of the SMC pulsars in the survey are discussed by Hong et al. (2016, in preparation). The data presented here were processed and 


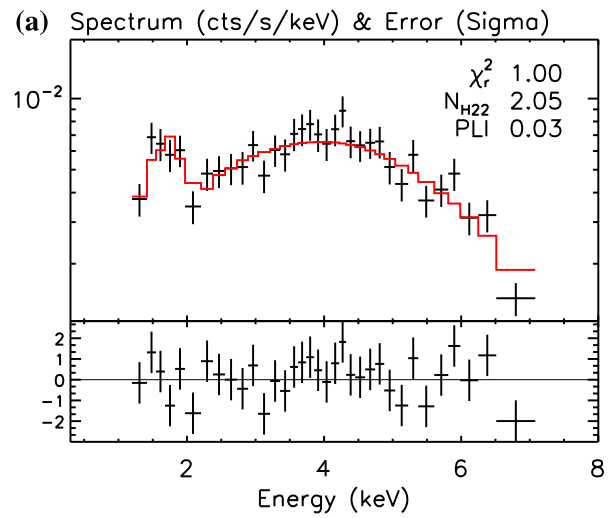

(d) Spectrum (cts/s/keV) \& Error (Sigma)

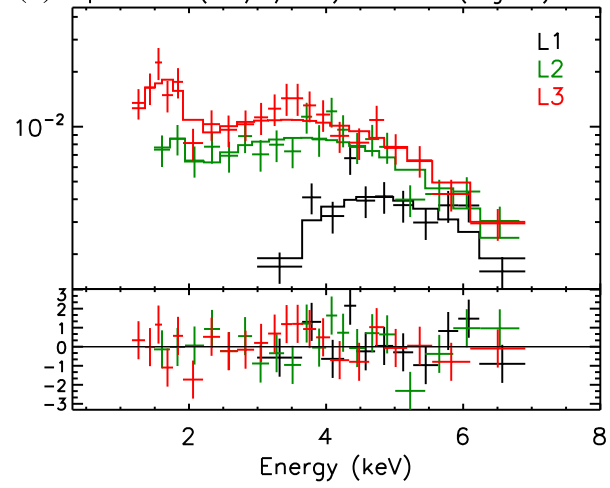

(b) Lightcurve (cts/ks)

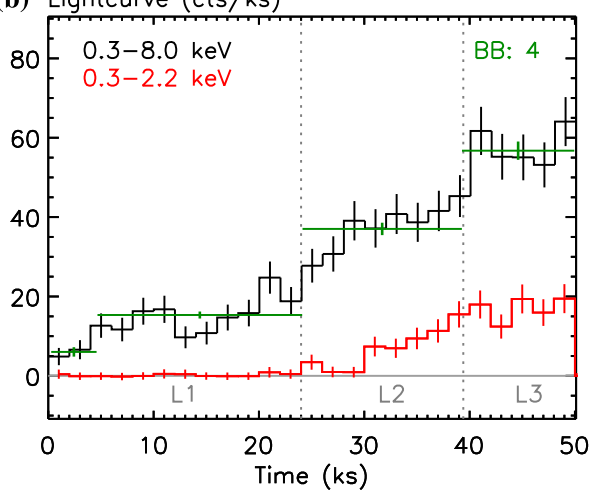

(e) Quartile Ratio

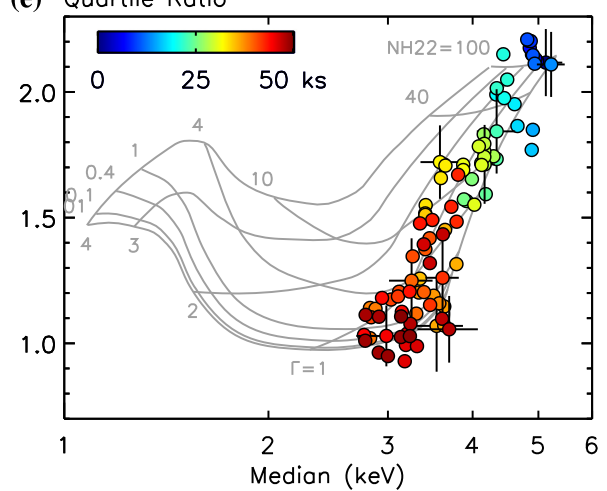

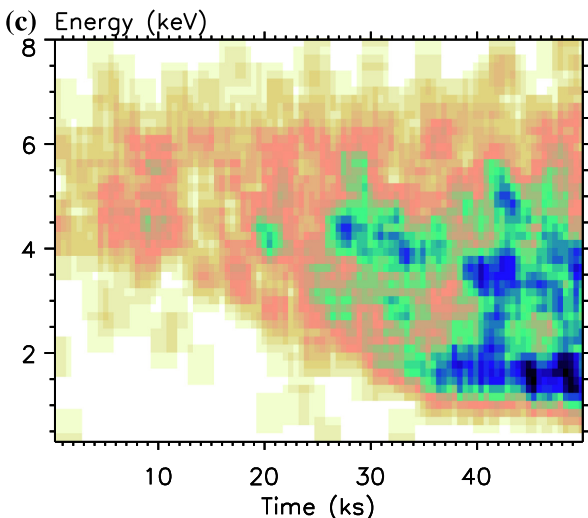

(f) $\mathrm{F}_{\mathrm{x}}\left(\times 10^{-13} \mathrm{erg} \mathrm{s}^{-1} \mathrm{~cm}^{-2}\right)$

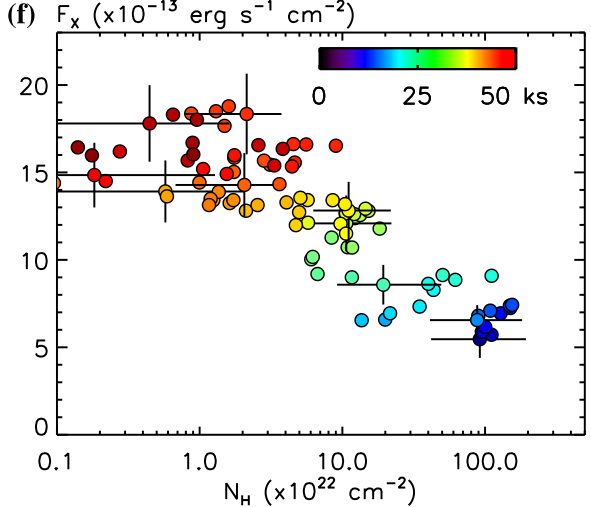

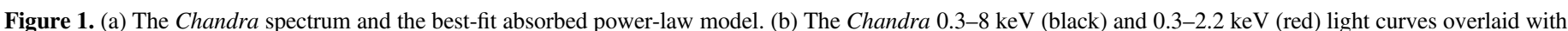

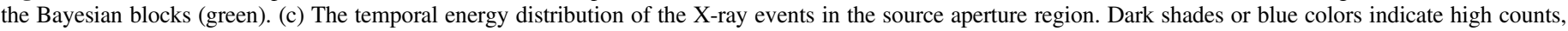

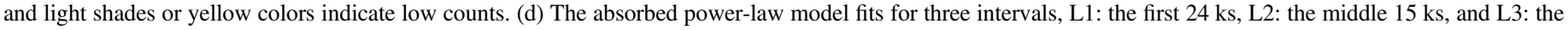

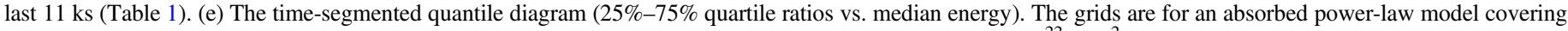

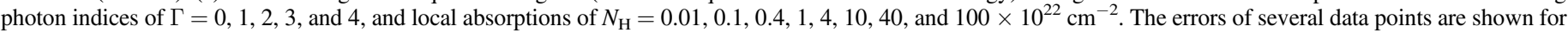

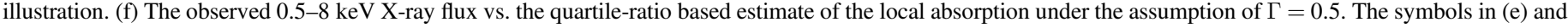
(f) are color-coded with the elapsed observing time.

analyzed by the latest X-ray analysis pipeline using CIAO version 4.6 developed for the Chandra Multi-wavelength Plane survey (Grindlay et al. 2005; Hong 2012).

The source was observed $\sim 7 ! 5$ off the aimpoint in both observations. The source was detected in the first observation with 1511 net counts in the $0.3-8 \mathrm{keV}$ band or a $0.5-8 \mathrm{keV}$ $\mathrm{X}$-ray luminosity of $4.7 \times 10^{35} \mathrm{erg} \mathrm{s}^{-1}$ at $60 \mathrm{kpc}$ (see below for the spectral model used), while it was not detected in the the second observation with an upper limit of 12 net counts at $3 \sigma$ significance $\left(L_{\mathrm{X}} \lesssim 4 \times 10^{33} \mathrm{erg} \mathrm{s}^{-1}\right)$. According to the Chandra archival data, a Chandra observation in 2002 October (Obs. ID 2945) also covered SXP 214 at $\sim 17^{\prime}$ off the aimpoint for $12 \mathrm{ks}$, but it was not detected $\left(L_{\mathrm{X}} \lesssim 3 \times 10^{34} \mathrm{erg} \mathrm{s}^{-1}\right)$. The following analysis is based on the 2013 January observation (Obs. ID 14670). The Chandra position of the source is R. A. $=00^{\mathrm{h}} 50^{\mathrm{m}} 11^{\mathrm{s}} .26$ and decl. $=-73^{\circ} 00^{\prime} 25^{\prime \prime} 6(\mathrm{~J} 2000)$ with a $95 \%$ error radius of 0.47 , where the error is based on the formula by Hong et al. (2005).

\section{SPECTRAL AND TEMPORAL EVOLUTION}

Figure 1(a) shows the overall X-ray spectrum and the best spectral fit using an absorbed power-law model with two absorption components: the Galactic foreground absorption fixed at $N_{\mathrm{H}}=6 \times 10^{20} \mathrm{~cm}^{-2}$ (Dickey \& Lockman 1990) with solar abundances $\left(Z=Z_{\odot}\right)$, and the free SMC and local absorption with reduced metal abundances of $Z=0.2 Z_{\odot}$ (Russell \& Dopita 1992) following the absorption model by
Wilms et al. (2000). Each spectral bin is set to contain at least 40 net counts. The best-fit photon index is $\Gamma=0.0 \pm 0.1$ with $N_{\mathrm{H}}=2.1 \times 10^{22} \mathrm{~cm}^{-2}$ and reduced $\chi^{2}=1$ (Table 1). An absorbed blackbody, thermal bremsstrahlung, or APEC model fits the X-ray spectrum poorly (reduced $\chi^{2}=1.4-4$ ).

The X-ray light curve of the source (Figure 1(b)) shows a steady rise with an average count rate of 30 counts $\mathrm{ks}^{-1}$. Bayesian block analysis, which searches for the change points between time intervals of statistically different rates (Scargle et al. 2013), identified four separate blocks (green). Figure 1(c) visualizes a remarkable change in the spectrum using the temporal energy distribution of the X-ray events in the source aperture region. The early part of the X-ray spectrum is limited to hard X-ray photons above $\sim 3 \mathrm{keV}$, and the softer X-ray photons below $2 \mathrm{keV}$ appeared only after $\sim 20 \mathrm{ks}$ into the observation.

To quantify the spectral evolution we divide the data set into three time intervals according to the Bayesian blocks: L1, L2, and L3 for the first $24 \mathrm{ks}$ (combining the first two blocks with relatively low counts), the middle $15 \mathrm{ks}$, and the last $11 \mathrm{ks}$, respectively. Figure 1(d) shows the best-fit joint spectral model of the three intervals with a common photon index. Each spectral bin of the segmented data set is set to contain at least 20-30 net counts. Table 1 lists the best-fit parameters for both joint and individual fits, which are all consistent with $\Gamma=0.5$, indicating that the spectral changes are driven by the variation in the local absorption. 
Table 1

Spectral Analysis Using an Absorbed Power-law Model

\begin{tabular}{|c|c|c|c|c|c|c|c|c|c|}
\hline \multirow{3}{*}{$\begin{array}{c}(1) \\
\text { Data Segment }\end{array}$} & \multirow{3}{*}{$\begin{array}{c}(2) \\
\text { Net Count } \\
0.3-8 \mathrm{keV}\end{array}$} & \multirow{3}{*}{$\begin{array}{l}(3) \\
\Gamma\end{array}$} & (4) & (5) & \multirow{3}{*}{$\begin{array}{c}(6) \\
\chi_{r}^{2} / \mathrm{DOF}\end{array}$} & (7) & (8) & (9) & $(10)$ \\
\hline & & & \multicolumn{2}{|c|}{$N_{\mathrm{H}}$ in $10^{22} \mathrm{~cm}^{-2}$} & & \multicolumn{2}{|c|}{$F_{\mathrm{X}}$} & \multicolumn{2}{|c|}{$L_{\mathrm{X}}$} \\
\hline & & & Joint Fit & (Indiv. Fit) & & Observed & Intrinsic & Observed & Intrinsic \\
\hline \multicolumn{2}{|c|}{ By Elapsed Time (Figure 1(d)) } & $0.5_{-0.1}^{+0.1}$ & & & $0.90 / 46$ & & & & \\
\hline 0-24 ks (L1) & $326 \pm 18$ & $\left(1.2_{-1.0}^{+1.1}\right)$ & $70.3_{-10.9}^{+11.6}$ & $\left(91.9_{-35.3}^{+38.3}\right)$ & $(1.43 / 8)$ & $7.0_{-1.8}^{+2.4}$ & $14.0_{-3.5}^{+4.7}$ & $3.0_{-0.8}^{+1.0}$ & $6.0_{-1.5}^{+2.0}$ \\
\hline \multicolumn{2}{|c|}{ By Folded Phases (Figure 2(c)) } & $0.2_{-0.1}^{+0.1}$ & & & $1.10 / 40$ & & & & \\
\hline $0.00-0.45(\mathrm{P} 1)$ & $224 \pm 15$ & $\left(-1.3_{-0.3}^{+0.7}\right)$ & $29.0_{-7.8}^{+9.2}$ & $\left(0.0_{-0.0}^{+14.1}\right)$ & $(0.59 / 6)$ & $4.5_{-1.0}^{+1.4}$ & $6.3_{-1.5}^{+2.0}$ & $1.9_{-0.4}^{+0.6}$ & $2.7_{-0.6}^{+0.8}$ \\
\hline $0.45-0.75(\mathrm{P} 2)$ & $646 \pm 25$ & $\left(0.5_{-0.2}^{+0.2}\right)$ & $3.2_{-1.2}^{+1.2}$ & $\left(5.0_{-1.6}^{+1.7}\right)$ & $(1.06 / 16)$ & $14.8_{-3.5}^{+4.6}$ & $16.2_{-3.8}^{+5.1}$ & $6.4_{-1.5}^{+2.0}$ & $7.0_{-1.6}^{+2.2}$ \\
\hline $0.75-1.00(\mathrm{P} 3)$ & $641 \pm 25$ & $\left(0.1_{-0.1}^{+0.2}\right)$ & $1.4_{-1.0}^{+1.0}$ & $\left(0.4_{-0.0}^{+1.3}\right)$ & $(1.07 / 16)$ & $16.3_{-3.8}^{+5.1}$ & $17.2_{-4.0}^{+5.4}$ & $7.0_{-1.6}^{+2.2}$ & $7.4_{-1.7}^{+2.3}$ \\
\hline
\end{tabular}

Notes. (3) The best-fit photon index, and (4) and (5) the SMC local absorption with the metal abundances of $Z=0.2 Z$ following Wilms et al. (2000) for an absorbed power-law model. The fit parameters in the parentheses are from individual spectral fits instead of joint fits. (6) The reduced $\chi^{2}$ and the degree of freedom (DOF). (7) The absorbed and (8) unabsorbed $0.5-8 \mathrm{keV}$ energy fluxes in $10^{-13} \mathrm{erg} \mathrm{s}^{-1} \mathrm{~cm}^{-2}$. (9) The absorbed and (10) unabsorbed $0.5-8 \mathrm{keV}$ luminosities at $60 \mathrm{kpc}$ in $10^{35} \mathrm{erg} \mathrm{s}^{-1}$. Errors in the table represent the $68 \%$ confidence (or $\pm 1 \sigma$ equivalent) interval.

Figure 1(e) illustrates the evolution of spectral parameters in a finer timescale using the energy quantile diagram (quartile ratio versus median energy), which can classify diverse spectral types without spectral bias often inherent to X-ray hardness or X-ray color-color diagrams (Hong et al. 2004). Each data point contains about 75 net counts in sliding time windows. The grids are for an absorbed power-law model covering a wide range of photon indices $(\Gamma=0-4)$ and local absorptions $\left(N_{\mathrm{H}}=10^{20-24} \mathrm{~cm}^{-2}\right)$. The rise in the count rate from $\sim 5$ to $60 \mathrm{cts} \mathrm{ks}^{-1}$ is well correlated with the significant reduction in the local absorption from $N_{\mathrm{H}} \sim$ $10^{24}$ to $10^{20} \mathrm{~cm}^{-2}$ for the aforementioned absorbed power-law model, whereas the photon index remains more or less constant. Figure 1(f) shows the correlation between the observed $0.5-8 \mathrm{keV}$ $\mathrm{X}$-ray flux and the local absorption, assuming $\Gamma=0.5$, whereas the intrinsic X-ray flux or luminosity remains more or less constant within the uncertainty (Table 1).

\section{TIMING ANALYSIS}

We search for periodic X-ray modulations in the $0.3-8 \mathrm{keV}$ light curve using a Lomb-Scargle (LS) periodogram (Scargle 1982). For the details of the timing analysis see Hong et al. (2016, in preparation). Figure 2(a) plots the LS periodogram over the entire search range, revealing a sharp peak at $211.5 \mathrm{~s}$. An increase in the power of the periodogram at periods longer than $10 \mathrm{ks}$ is due to the long-term rise of the light curve. We refine the modulation period with a follow-up search using the epoch folding (EF; Leahy et al. 1983) and LS methods around the initial period. Both the EF and LS periodograms peak at the same period of $211.49 \pm 0.42 \mathrm{~s}$ (Figure 2(b)). The error of the modulation period is a $1 \sigma$-equivalent width $(34 \%)$ of the peak in the periodogram, which tends to be a bit conservative, relative to the error estimate $(0.13 \mathrm{~s})$ based on Horne \& Baliunas (1986). Nonetheless, the newly detected pulse period is not only significantly shorter than the previously reported period of $214.045 \pm 0.052 \mathrm{~s}$ by $\mathrm{C} 11$ from the XMMNewton observation in 2009 November, but it is also against the long-term spin-down trend $\left(\dot{P} \sim+0.1 \mathrm{~s} \mathrm{yr}^{-1}\right.$ with the average period of $213.7 \pm 0.1 \mathrm{~s}$ ) measured by RXTE (Klus et al. 2014, hereafter K14). On the other hand, the individual period measurements by K14 show a large scatter ranging from 211 to $216 \mathrm{~s}$, and two out of 17 RXTE measurements show the periods consistent with our result.

Figure 2(d) illustrates the drastic spectral variation with pulse phase using the energy versus phase distribution of the X-ray events. The energy-band selected pulse profiles in Figure 2(e) show large peak-to-valley amplitudes $\left(A_{\bmod } \geqslant 95 \%\right)$ and high pulse fractions $\left(P_{F} \geqslant 91 \%\right),{ }^{13}$ which contrasts with the somewhat marginal $P_{F}$ of $29 \%$ measured by XMMNewton (C11). The Chandra pulse profile shows clear onand off-phases of almost equal durations. The soft X-ray emission below $2 \mathrm{keV}$ appears fully "eclipsed" during the offphase and exhibits a two-step rise during the on-phase, whereas the hard X-ray emission is more or less uniform during the onphase with some trailing into the off-phase.

We divide the pulse phases into three segments based on the soft-band pulse profile: P1 for the off-phase covering $0.00-0.45$, P2 for the first part of the rise covering 0.45-0.75, and P3 for the peak covering 0.75-1.00. Figure 2(c) shows the best-fit joint model of the three segments with a common photon index, and Table 1 lists the best-fit parameters for both joint and individual fits. When $\Gamma \lesssim 0$ (P1), the photon index becomes somewhat degenerate with the absorption, so the best-fit parameters can be misleading. On the other hand, in the joint fit and the phaseresolved quantile diagram in Figure 2(f), there appears to be a clear trend that the spectral evolution over pulse cycles is also dominated by the changes in the local absorption. At the peak of the pulse profile (phase $\sim 0.9$ ), the local absorption drops below $N_{\mathrm{H}}=10^{20} \mathrm{~cm}^{-2}$, whereas during the off-phases $(\sim 0.1-0.5)$, it rises well above $10^{23} \mathrm{~cm}^{-2}$. The quantile diagram in Figure 2(i) compares the spectral types of the three phases of each time interval. The spectral evolution over the pulse phase within each interval is not dramatic, indicating that the spectral evolution seen in Figures 2(c) and (f) is likely amplified by the temporal evolution.

\footnotetext{
$13 A_{\text {mod }}$ is defined as $1-r_{\min } / r_{\max }$, where $\mathrm{r}^{\mathrm{m}}$ in and $r_{\max }$ are the minimum and maximum of the folded light curve, respectively. $P_{F}$ is defined as the ratio of the pulsating flux above the minimum to the total flux, i.e., $\Sigma_{i}\left(r_{i}-r_{\min }\right) / \Sigma_{i} r_{i}$, where $r_{i}$ is the rate of the folded bin, $i$.
} 

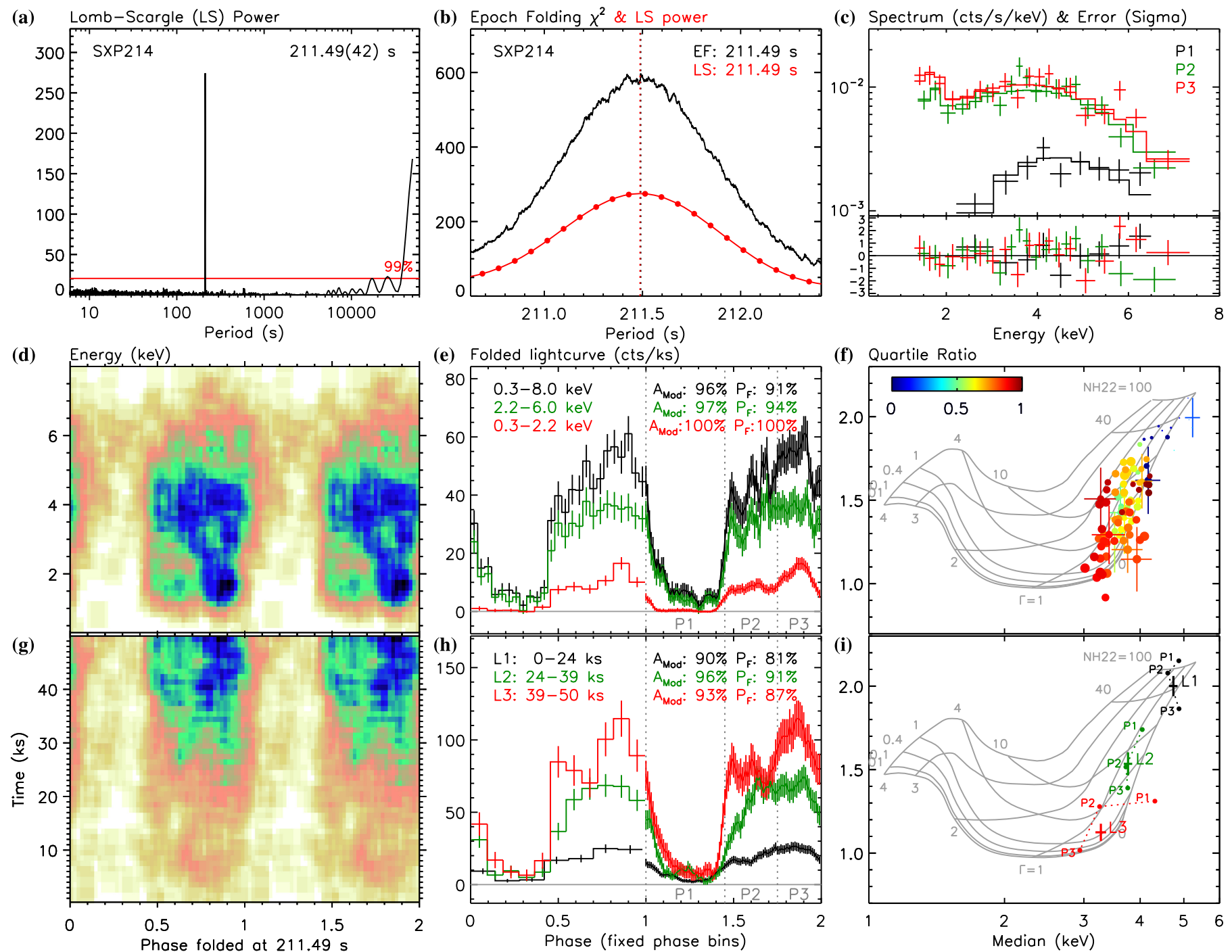

Figure 2. (a) The Lomb-Scargle periodogram with the $99 \%$ confidence level. (b) A close-up of the epoch folding and Lomb-Scargle periodograms around the pulse period. (c) The absorbed power-law model fits for three phase segments, P1: 0.00-0.45, P2: 0.45-0.75, and P3: 0.75-1.00. (d) The energy vs. pulse phase distribution of the X-ray events. (e) The folded light curves in the $0.3-2.2 \mathrm{keV}$ (red), 2.2-6.0 keV (green) and 0.3-8.0 keV (black) bands using fixed-size phase bins (phases 0-1) and sliding window bins (phases 1-2). (f) The phase-resolved quantile diagram. The symbol colors match the pulse phase, and the symbol sizes are proportional to the count rates. (g) The time vs. pulse phase distribution of the X-ray events. (h) The $0.3-8 \mathrm{keV}$ folded light curves of the three time intervals, L1, L2, and L3. (i) The quantile diagram for the three phase segments of each interval.

Figure 2(g) shows the time versus pulse phase distribution of the X-ray events. The pulsation is present from the very beginning of the observation when no soft X-ray emission below $2 \mathrm{keV}$ was observed. The apparent skewness in the distribution (e.g., at phase $\sim 0$ ), which is usually an indication of an inaccurate period estimate (Hong et al. 2016, in preparation), is an artifact due to the flux change with the time: i.e., the distribution, if renormalized at each time bin, would not be skewed (not shown). Figure 2(h) shows the folded light curve of each time interval, and the strong pulsation is observed in all three intervals $\left(A_{\bmod } \geqslant 90 \%\right)$.

\section{OGLE LIGHT-CURVE ANALYSIS}

Figure 3(a) shows the optical light curve in I mag spanning over 20 years using the data from the OGLE-II, III, and IV surveys. The red circles and blue diamonds mark the three Chandra and four XMM-Newton observations, respectively. The X-ray pulsations (solid symbols) were detected when the optical light curve was experiencing a significant drop or recovering from the drop. RXTE also detected the X-ray pulsations from the source multiple times (purple arrows; K14).

Given the uncertainty in the orbital period of the system, we search for periodic modulation in the full OGLE light curve using the LS periodogram. The LS periodogram of the raw light curve shows three dominant periods of $0.5,1$, and 348 days. Detrending techniques eliminate these false periods to varying degrees, but the final periodicity results are not sensitive to the detrending techniques. We use the moving average of 20 data points as the light curve trend.

We also calculate the LS periodogram of the OGLE light curve using sliding windows of 500 days to identify any changes in the periodicity. Indeed, the periodicity transitions in about MJD 55000, and then the last 500 days of the light curve do not show any clear sign of periodicity. In the first 5000 days of the light curve, 0.82 and 4.58 day periods are most dominant as reported by C11 and Schmidtke \& Cowley (2011). We note that the 0.82 day period is a beat period among $0.5,1$, and 4.58 
(a)

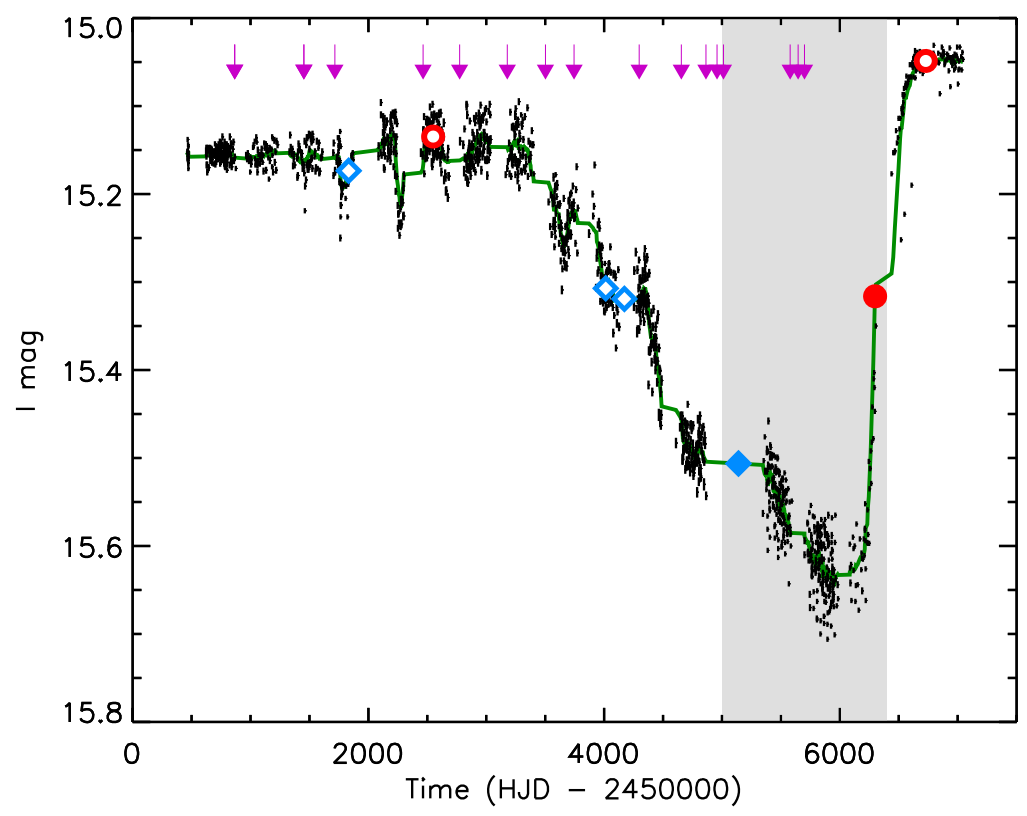

(b)

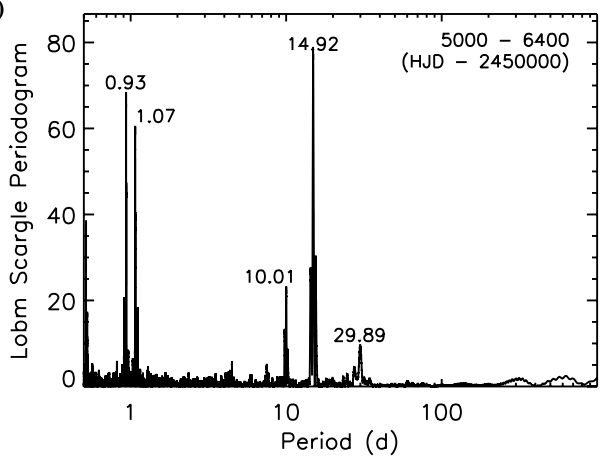

(c)

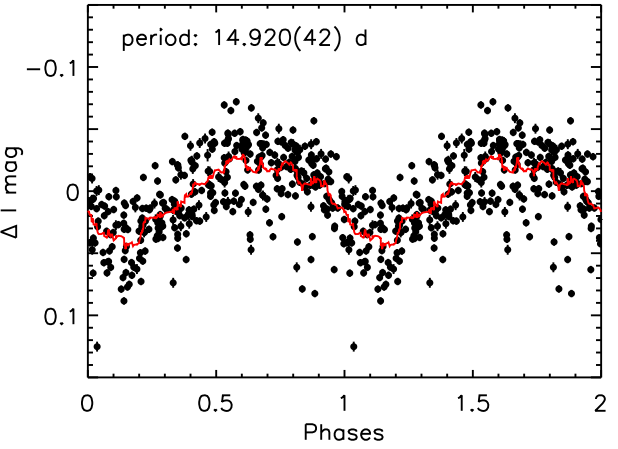

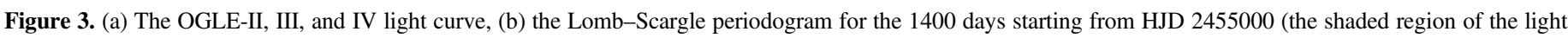

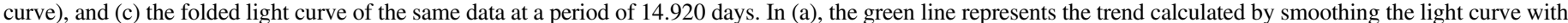

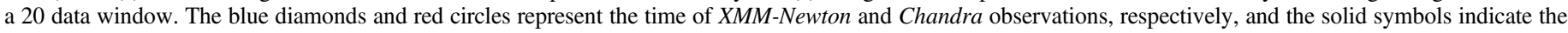
detection of the X-ray periodicity. The pulsation detections by RXTE are marked by the magenta arrows.

day periods. Figure 3(b) shows the periodogram of the next 1400 days where a 14.92 day period is most prominent. Its second harmonic (29.89 days), which is consistent with the 29.91 day period reported by Schmidtke et al. (2013), is marginally significant. The 0.93 and 1.07 day periods are the beating periods among 0.5, 1, and 14.92 days. Figure 3(c) shows the folded light curve at the 14.92 day period. The analysis of the full OGLE light curve did not reveal any other new periods. Given their instability, it appears premature to claim that any of the above periods is the orbital period

\section{DISCUSSION}

The typical orbital period of Be-XRBs with $\sim 200-300 \mathrm{~s}$ pulsations is $\sim 30-200$ days based on the so-called "Corbet" diagram (e.g., Reig 2007; Cheng et al. 2014). K14 assumed that 4.58 days as the orbital period of SXP 214, but according to their own orbital versus spin period diagram, SXP 214 is an outlier and 4.58 days appears too short for the orbital period. C11 detected weak $\mathrm{H} \alpha$ emission (equivalent width: $-1.5 \pm 1.0 \AA$ ) on MJD 55716 when the optical flux was near its historical minimum. Since it is recognized that the continuum variations in Be-stars trace the size of the circumstellar disk (e.g., Reig 2011), the 14.92 or 29.89 day periods, measured when the disk was relatively small, may represent the true orbital period. The observed historical maximum X-ray luminosity is less than $6 \times 10^{36} \mathrm{erg} \mathrm{s}^{-1}(\mathrm{~K} 14){ }^{14}$ In fact, the system was detected only in two out of seven

\footnotetext{
${ }^{14} \mathrm{~K} 14$ assumed $P_{F}=33 \%$ when converting the RXTE counts to the luminosity. Their luminosity value can be an overestimate by a factor of three if the pulsed fraction is close to $100 \%$, as seen in this analysis. On the other hand, K14 assumed $\Gamma=1.5$ for the spectral model, which can lower their luminosity estimates by $\sim 30 \%$ for a harder spectrum with $\Gamma=0.5$. Combining these two, the luminosity estimated by K14 for SXP 214 can be an overestimate by about a factor of two.
}

observations (three with Chandra and four with XMM-Newton), which is about $30 \%$ of the duty cycle. According to Reig (2007), these findings suggest that SXP 214 is in a highly eccentric orbit $(\gtrsim 0.3)$ with a long orbital period ( $~ 20$ days) and should exhibit Type I bursts $\left(<10^{37} \mathrm{erg} \mathrm{s}^{-1}\right)$. They further suggest that the circumstellar disk is likely only mildly perturbed (but not fully truncated) by the NS.

Our measurement of the spin period indicates that the system may have gone through sudden spin-up episodes recently. In a system with a highly elliptical orbit, accretion-induced spin-up episodes are likely to occur regularly, often near periastron passages. The orbital speed $(v)$ of the NS is

$$
v=\left[\frac{2 \pi G\left(M_{1}+M_{2}\right)}{P_{\mathrm{o}}}\right]^{1 / 3}\left[\frac{1+2 \epsilon \cos (\phi)+\epsilon^{2}}{1-\epsilon^{2}}\right]^{1 / 2},
$$

where $G, M_{1}, M_{2}, \epsilon, \phi$, and $P_{\mathrm{o}}$ are the gravitational constant, the masses of the primary and the NSs, the eccentricity, the orbital phase, and the orbital period, respectively. Within about $120^{\circ}$ of the periapsis (i.e., $|\phi| \lesssim 120^{\circ}$ ) which covers about $30 \%$ of the orbital period, for $\epsilon=0.5, M_{1}=10 M_{\odot}$, and $M_{2}=1.4$ $M_{\odot}, v \sim 150-270\left(M_{11.4} / P_{30}\right)^{1 / 3} \mathrm{~km} \mathrm{~s}^{-1}$ or $150-270 a_{0.43} P_{30}^{-1}$ $\mathrm{km} \mathrm{s}^{-1}$, where $M_{11.4}$ is the combined mass in units of $11.4 M_{\odot}$, $P_{30}$ is $P_{0}$ in units of 30 days, and $a_{0.43}$ is the semimajor axis in units of $0.43 \mathrm{AU}$. Therefore, the apparent change in the spin period due to the orbital motion-induced Doppler effect is less than $\sim 0.11-0.19\left(M_{11.4} / P_{30}\right)^{1 / 3} \mathrm{~s}$ for $\epsilon=0.5$, which is too small to account for the observed period difference.

According to Cheng et al. (2014), accretion through a disk can be an efficient spin-up process for the NS, whereas quasispherical or advection dominated accretion (Narayan \& Yi $1994,1995)$ is relatively inefficient. Even in the case that the 
accretion is always through a disk, if the accretion disk around the pulsar is frequently reformed near periastron passages due to a highly eccentric orbit, it is not surprising to see the spin-up and spin-down torque reversal depending on whether each newly formed disk is prograde or retrograde relative to the NS spin (Nelson et al. 1997). Given the long-term spin-down trend, even with the two earlier RXTE measurements showing similarly short periods (K14), the spin-up and spin-down torque reversal may occur routinely in SXP 214 in addition to the relatively slow spin-down trend due to the rotational energy loss when there is no accretion.

Roughly two years have passed between the last measurement of the spin period by K14 and our measurements. Indeed, the last few measurements of the spin period by K14 already show a possible spin-up trend. If we assume that the recent spin-up trend has lasted $\sim 1-3$ years, then $-\dot{P} \sim 0.56-1.7 \mathrm{~s} \mathrm{yr}^{-1}$. For the accretion torque-induced spin-up model by Ghosh \& Lamb (1979) under the assumption that the observed peak luminosity of $\sim 10^{36} \mathrm{erg} \mathrm{s}^{-1}$ is due to the accretion, the only possible solution for an NS with a mass of $M=1.4 M_{\odot}$ and a radius of $R=10^{6} \mathrm{~cm}$ is with $\dot{P} \sim-0.48 \mathrm{~s} \mathrm{yr}^{-1}$ and a magnetic field of $B \sim$ $10^{13} \mathrm{G}$. The matching accretion rate is $\dot{M} \sim 5 \times 10^{15} \mathrm{~g} \mathrm{~s}^{-1}$ or $8 \times 10^{-11} M_{\odot} \mathrm{yr}^{-1}$. The observed luminosity and the accretion rate are a bit low for the estimated spin-up rate, even with a strong $B$ field, especially considering that the X-ray emission and the spin-up trend have likely been episodic. If we use the historical average of the X-ray luminosities measured by $R X T E(\sim 3 \times$ $10^{36} \mathrm{erg} \mathrm{s}^{-1}$, K14), a solution for the model by Ghosh \& Lamb (1979) is available for the full range of $-\dot{P} \sim 0.56-1.7 \mathrm{~s} \mathrm{yr}^{-1}$, with $B \sim(0.03-1.5) \times 10^{12} \mathrm{G}$ and $\dot{M} \sim 5.1 \times 10^{-10} M_{\odot} \mathrm{yr}^{-1}$. With the duty cycle of $\sim 30 \%$, the actual spin-up rate during the accretion has to be about three times higher, which effectively means $-\dot{P} \sim 1.9-5.7 \mathrm{~s} \mathrm{yr}^{-1}$ during the accretion. The solution for the model by Ghosh \& Lamb (1979) exists for a partial range of $-\dot{P} \sim 1.9-4.8 \mathrm{~s} \mathrm{yr}^{-1}$ with $B \sim(0.44-29) \times 10^{12} \mathrm{G}$ and $\dot{M} \sim 8.4 \times$ $10^{-10} M_{\odot} \mathrm{yr}^{-1}$ during the accretion (or $2.5 \times 10^{-10} M_{\odot} \mathrm{yr}^{-1}$ on average).

The anti-correlation between the observed flux and the absorption during pulsation as shown in Figure 2(f) suggests that the pulsation might be modulated with the periodic occultation of the emission region by an intervening absorber (e.g., Suchy et al. 2008). The modulated absorption, however, cannot explain the missing soft X-ray component during the early time of the observation when the strong pulsation was present (Figures 2(g) and (h)). This implies that, in the beginning, the pulsar must have been inside or behind a heavy absorber independent of the pulsation geometry. A natural candidate for such an absorber is the circumstellar disk of the companion. The soft X-ray emission at the later time, then, indicates that the NS came out of the circumstellar disk and into view without obscuration by the disk. Coe et al. (2015) showed that a similar picture can explain the orbital eclipse in the longterm X-ray light curve of SXP 5.05. This analysis demonstrates, for the first time, the changes in the X-ray emission as the NS crosses the circumstellar disk.

Alternatively, one can consider accretion wake or clumpy winds as an absorber. The stellar wind from a typical B2-B3 star, however, is not strong enough to trigger an accretion wake or to generate clumpy winds. For instance, clumpy winds in $4 \mathrm{U}$ 1700-37, which cause an absorption of $\sim 10^{24} \mathrm{~cm}^{-1}$, are from an O star with $\dot{M} \sim 10^{-5}-10^{-6} M_{\odot} \mathrm{yr}^{-1}$ (Haberl et al. 1989),

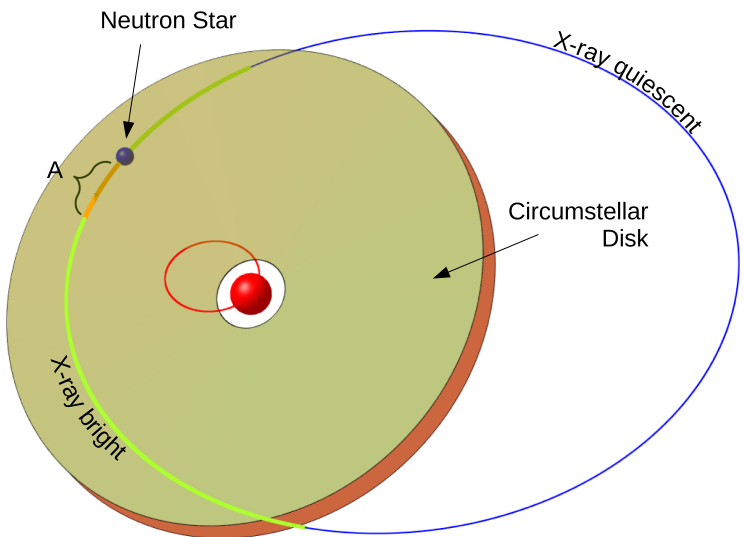

Figure 4. A possible orbital configuration of SXP 214 under the assumptions of $M_{1} \sim 10 M_{\odot}, M_{2} \sim 1.4 M_{\odot}, P_{\mathrm{o}} \sim 30$ days, and $\delta \sim 15^{\circ}$. For about $25 \%$ of the orbital period the NS is X-ray bright, which is marked with the thicker (green) line. The interval marked "A" (orange line) represents a possible observing period for Obs. ID 14670. The red ellipse represents the orbit of the primary star.

whereas the mass loss $(\dot{M})$ for B0-B1 stars is already $\lesssim 10^{-9.5} M_{\odot} \mathrm{yr}^{-1}$ (Smith 2014).

In fact, we have likely witnessed the NS crossing through (instead of behind) the circumstellar disk. The observed spin-up trend and associated torque reversal favor the accretion through a disk instead of quasi-spherical winds. The constant intrinsic $\mathrm{X}$-ray luminosity in Table 1 indicates that the accretion was in a steady state during our observation. This appears to favor the NS crossing behind the circumstellar disk without contact during our observation. But then it implies a relatively long lifetime of the accretion disk and a sharply truncated circumstellar disk for the NS to emerge in a short time ( $\lesssim 30 \mathrm{ks})$. Feeding enough material for the lasting accretion disk would likely require a circumstellar disk with a high density, which in turn would lead to a high peak X-ray luminosity ( $>10^{37} \mathrm{erg} \mathrm{s}^{-1}$; e.g., SXP5.05) and a long emission duty cycle, at odds with the long-term properties of SXP 214.

On the contrary, the observed rare episodic X-ray emission and the likely long orbital period suggest a relatively short lifetime of the accretion disk and an only mildly perturbed circumstellar disk. A relatively low X-ray luminosity also implies that the NS crosses a low density region of the circumstellar disk (see below). The relatively low optical emission (Figure 3(a)) during our observation also implies a small circumstellar disk. Thus, it is plausible that the accretion disk became stable while feeding through the circumstellar disk, and the intrinsic X-ray luminosity remained constant for a while after the NS just emerged from the circumstellar disk. Therefore, the NS crossing through the circumstellar disk provides a more consistent picture.

Figure 4 illustrates a possible orbital configuration of SXP 214 under the assumptions of $M_{1} \sim 10 M_{\odot}, M_{2} \sim 1.4 M_{\odot}, P_{\mathrm{o}} \sim$ 30 days. The radius of the circumstellar disk shown in the figure is about $12 R_{*} \sim 60 R_{\odot}$, and an inclination angle $(\delta)$ between the disk and the orbital plane is about $15^{\circ}$. In this picture, the NS crosses the circumstellar disk near the periastron passage once per orbit and for about $25 \%$ of the orbital period (depending on the orbital motion induced change in the disk, e.g., warping), the first Lagrangian point of the NS is on contact with the circumstellar disk, during which the NS is potentially X-ray bright (thicker green line). The interval marked by " $\mathrm{A}$ " (orange line) can be the $50 \mathrm{ks}$ observing period 
of Obs. ID 14670, where the NS emerges from the disk at about 20-30 ks into the observation.

While the above illustration provides a possible picture consistent with the observation including the X-ray emission duty cycle, note how the accretion phase remains relatively short is not clearly understood. In particular, a caveat in the accretion disk for Type I burst systems is that the viscous timescale of a standard thin $\alpha$ disk (Shakura \& Sunyaev 1873) is expected to be longer than the orbital period, which should produce a somewhat persistent X-ray emission. Okazaki et al. (2013) invokes a radiatively inefficient accretion flow to explain the relatively short X-ray emission duty cycle for TypeI burst systems, where the accretion flow is X-ray faint and the observed X-ray is mainly from the radiation at the poles. Relatively high modulation amplitude $(\gtrsim 90 \%)$ indeed suggests that the observed X-ray emission from SXP 214 is from the poles, but the exact accretion flow around the NS remains uncertain.

In our observation the soft X-ray component was hidden for at least $20 \mathrm{ks}$. Assuming the NS was already inside of the circumstellar disk in the beginning of the observation based on the constant intrinsic luminosity and the orbital path is locally straight, we can place a limit on the height $(H)$ of the circumstellar disk using the travel distance of the NS in $20 \mathrm{ks}$. For an inclination angle $(\delta)$ between the disk and the orbital plane,

$$
H \gtrsim(3.1-5.3) \times 10^{6}\left(M_{11.4} / P_{30}\right)^{1 / 3} \sin (\delta) \mathrm{km} .
$$

If the majority of the observed extinction $\left(\sim 10^{24} \mathrm{~cm}^{-2}\right)$ at the early times is due to a simple slab-shape disk of a uniform density, ${ }^{15}$ the density $(\rho)$ of the circumstellar disk is

$\rho \lesssim(3.1-5.4) \times 10^{-12}\left(P_{30} / M_{11.4}\right)^{1 / 3} \sin (\delta)^{-1} \sin (\theta) \mathrm{g} \mathrm{cm}^{-3}$,

where $\theta$ is the angle between the disk and the line of sight (i.e., $\theta=0$ for an edge-on view). The density of the circumstellar disk of Be-stars lies in the range between about $10^{-12}$ to a few times $10^{-10} \mathrm{~g} \mathrm{~cm}^{-3}$ (Rivinius et al. 2013) and the disks of BeXRBs are about 1.5 times denser (Reig et al. 2016), so the above estimate for SXP 214 indicates that the section the NS crosses is of relatively low density.

The mass the NS accretes can be estimated using the volume swept by the NS under the Bondi accretion.

$$
\dot{M} \sim 4 \pi \eta \rho \frac{G^{2} M_{2}^{2}}{v_{r}^{3}} \frac{H}{v},
$$

where $v_{r}$ is the orbital speed of the NS relative to the gas in the circumstellar disk, and $\eta$ is the accretion fraction relative to the circumstellar disk mass the NS disrupts as it crosses. Assuming the velocity of the gas in the circumstellar disk is in the same order of the NS' orbital speed $v, v_{r} \sim \sqrt{2} v$. Therefore,

$$
\begin{aligned}
\dot{M} & \sim(4.5-50) \times 10^{23} M_{1.4}^{2}\left(P_{30} / M_{11.4}\right)^{4 / 3} \sin (\theta) \text { g per passage } \\
& =(3.1-28) \times 10^{-9} M_{1.4}^{2} P_{30}^{1 / 3} / M_{11.4}^{4 / 3} \sin (\theta) M_{\odot} \mathrm{yr}^{-1} .
\end{aligned}
$$

\footnotetext{
${ }^{15}$ We use a simple model, given many unknown system parameters. Along with a possible density profile in the circumstellar disk, the orbital motion of the primary star (Figure 4) can cause warping in the disk, generating an additional density variation.
}

If the NS captures about $1 \%-10 \%$ of the mass it disrupts (i.e., $\eta \sim 0.01-0.1)$, the accretion rate meets what is required for the observed spin-up process $\left(\sim 2.5 \times 10^{-10} M_{\odot} \mathrm{yr}^{-1}\right)$.

The spectral variation during the pulsation can be understood by the periodic occultation of the emission region due to the NS rather than a separate absorber. It is because the phase-resolved spectral analysis of each interval in Figure 2(i) shows that the local absorption is not likely the dominant factor for pulsation, and the apparent correlation between the absorption and the pulse phase seen in the full interval (Figures 2(c) and (f)) is likely the result of the temporal evolution. For instance, the trailing hard X-ray component during the ingress to the offphase can be due to Comptonized reflection from the accretion stream. Alternatively, the soft X-rays may be mainly from a halo around the bottom of the accretion column at the NS surface (e.g., Davidson \& Ostriker 1973; Lyubarskii \& Syunyaev 1988; Kraus et al. 2003), which can explain the total absence of the soft component during the off-phases in the pulse profile (red in Figure 2(e)). A circular emission spot on the surface of a pulsar can produce the observed eclipse-like feature in the pulse profile when combined with general relativistic effects (e.g., Wang \& Welter 1981).

The remarkable variation in the X-ray emission from SXP 214 indicates that the NS was caught while crossing the circumstellar disk of the companion Be-star. X-ray observations provide a rare opportunity to study the properties of the circumstellar disk and the emission geometry of the NS surface.

V.A. acknowledges financial support from NASA/Chandra grants GO3-14051X, AR4-15003X, NNX15AR30G, and NASA/ADAP grant NNX10AH47G. A.Z. acknowledges financial support from NASA/ADAP grant NNX12AN05G, and funding from the European Research Council under the European Union's Seventh Framework Programme (FP/2007-2013)/ERC Grant Agreement no. 617001. J.D., P.P., and T.G. acknowledge financial support from NASA contract NAS8-03060. M.S. acknowledges support by the the Deutsche Forschungsgemeinschaft through the Heisenberg Programme (SA 2131/3-1). S.L. acknowledges financial support from NASA/ADAP grant NNX14-AF77G. The OGLE project has received funding from the National Science Centre, Poland, Grant MAESTRO 2014/14/ A/ST9/00121 to AU. We thank the anonymous referee for helpful comments and suggestions.

\section{REFERENCES}

Cheng, Z.-Q., Shao, Y., \& Li, X.-D. 2014, ApJ, 786, 128

Coe, M. J., Bartlett, E. S., Bird, A. J., et al. 2015, MNRAS, 447, 2387

Coe, M. J., Haberl, F., Sturm, R., et al. 2011, MNRAS, 414, 3281 (C11)

Coe, M. J., \& Kirk, J. 2015, MNRAS, 452, 969

Davidson, K., \& Ostriker, J. P. 1973, ApJ, 179, 585

Dickey, J. M., \& Lockman, F. J. 1990, ARA\&A, 28, 215

Ghosh, P., \& Lamb, F. K. 1979, ApJ, 234, 296

Grindlay, J. E., Hong, J., Zhao, P., et al. 2005, ApJ, 635, 920

Haberl, F., \& Sturm, R. 2015, arXiv:1511.00445

Haberl, F., White, N. E., \& Kallman, T. R. 1989, ApJ, 343, 409

Hong, J. 2012, MNRAS, 427, 1633

Hong, J., Schlegel, E. M., \& Grindlay, J. E. 2004, ApJ, 614, 508

Hong, J., van den Berg, M., Schlegel, E. M., et al. 2005, ApJ, 635, 907

Horne, J. H., \& Baliunas, S. L. 1986, ApJ, 302, 757

Klus, H., Ho, W. C. G., Coe, M. J., Corbet, R. H. D., \& Townsend, L. J. 2014, MNRAS, 437, 3863 (K14)

Kraus, U., Zahn, C., Weth, C., \& Ruder, H. 2003, ApJ, 590, 424

Leahy, D. A., Darbro, W., Elsner, R. F., et al. 1983, ApJ, 266, 160

Lyubarskii, Y. E., \& Syunyaev, R. A. 1988, SvAL, 14, 390

Narayan, R., \& Yi, I. 1994, ApJL, 428, L13 
Narayan, R., \& Yi, I. 1995, ApJ, 452, 710

Nelson, R. W., Bildsten, L., Chakrabarty, D., et al. 1997, ApJL, 488, L117

Okazaki, A. T., Hayasaki, K., \& Moritani, Y. 2013, PASJ, 65, 41

Reig, P. 2007, MNRAS, 377, 867

Reig, P. 2011, Ap\&SS, 332, 1

Reig, P., Nersesian, A., Zezas, A., Gkouvelis, L., \& Coe, M. J. 2016, arXiv: 1603.08327

Rivinius, T., Carciofi, A. C., \& Martayan, C. 2013, A\&ARv, 21, 69

Russell, S. C., \& Dopita, M. A. 1992, ApJ, 384, 508

Scargle, J. D. 1982, ApJ, 263, 835
Scargle, J. D., Norris, J. P., Jackson, B., \& Chiang, J. 2013, ApJ, 764, 167 Schmidtke, P. C., \& Cowley, A. P. 2011, ATel, 3305, 1

Schmidtke, P. C., Cowley, A. P., \& Udalski, A. 2013, ATel, 4936, 1

Shakura, N. I., \& Sunyaev, R. A. 1973, A\&A, 24, 337

Smith, N. 2014, ARA\&A, 52, 487

Suchy, S., Pottschmidt, K., Wilms, J., et al. 2008, ApJ, 675, 1487

Townsend, L. J., Coe, M. J., Corbet, R. H. D., \& Hill, A. B. 2011, MNRAS, 416, 1556

Wang, Y.-M., \& Welter, G. L. 1981, A\&A, 102, 97

Wilms, J., Allen, A., \& McCray, R. 2000, ApJ, 542, 914 